Case

Report

\title{
A Case of an Acute Cervicomediastinal Hematoma Secondary to the Spontaneous Rupture of a Parathyroid Adenoma
}

\author{
Noriko Yoshimura, MD, ${ }^{1}$ Hidenori Mukaida, MD, PhD, ${ }^{1}$ Takeshi Mimura, MD, PhD ${ }^{2}$ \\ Kazuhiro Iwata, MD, PhD, ${ }^{3} \mathrm{Ai}$ Amioka, MD, ${ }^{1}$ Naoki Hirabayashi, MD, $\mathrm{PhD},{ }^{1}$ and \\ Wataru Takiyama, $\mathrm{MD}, \mathrm{PhD}^{1}$
}

\begin{abstract}
We herein report the case of a patient with an acute cervicomediastinal hematoma secondary to the spontaneous rupture of a parathyroid adenoma. A 47-year-old female presented with swelling and pain in the neck. She had no history of trauma or of having undergone any medical or odontological procedures. An ultrasound examination revealed the presence of an obscure mass located behind the right lobe of the thyroid gland. A computed tomography scan showed the presence of a low-density lesion extending from the retropharynx to the mediastinum, a high-density lesion located behind the right lobe of the thyroid gland and a right pleural effusion. Because the patient's neck swelling and anemia gradually worsened, she underwent emergency surgery. The neck was found to be swollen due to a hematoma; however, no abscesses were detected in the operative field. Thoracoscopy of the right chest showed no active bleeding. The fragmented mass was histopathologically diagnosed as a parathyroid adenoma with acute hemorrhage, which is quite rare. Our experience suggests that, in patients with severe cervicomediastinal hematomas without any trauma or trigger, a diagnosis of spontaneous rupture of a parathyroid gland lesion should be considered.
\end{abstract}

Keywords: cervicomediastinal hematoma, parathyroid adenoma, spontaneous rupture

\section{Introduction}

Acute cervicomediastinal hematomas caused by the spontaneous rupture of parathyroid adenomas are rare. In 1934, Capps ${ }^{1)}$ first described cervical and mediastinal

${ }^{1}$ Department of Surgery, Hiroshima City Asa Hospital, Hiroshima, Japan

${ }^{2}$ Department of Internal Medicine, University of Michigan, Michigan, USA

${ }^{3}$ Department of Otolaryngology, Hiroshima City Asa Hospital, Hiroshima, Japan

Received: June 5, 2012; Accepted: August 3, 2012

Corresponding author: Noriko Yoshimura, MD. Department of Surgery, Hiroshima City Asa Hospital, 2-1-1 Kabeminami, Asakita-ku, Hiroshima, Hiroshima 731-0293, Japan

Email: yoshimuranoriko32@hotmail.co.jp

(C)2014 The Editorial Committee of Annals of Thoracic and Car-

diovascular Surgery. All rights reserved. hemorrhages originating from hyperplastic parathyroid glands. This rare type of hematoma occurs abruptly without any preceding trauma or iatrogenic damage, making it very difficult to determine the cause precisely. Patients with cervicomediastinal hematomas require immediate treatment because the hemorrhages may lead to lifethreatening conditions, i.e. airway obstruction due to massive bleeding in the neck. We herein report a rare case of cervicomediastinal hematoma secondary to a spontaneous rupture of a parathyroid adenoma.

\section{Case report}

A 47-year-old female noticed swelling of the anterior neck for two days prior to experiencing painful swelling of the neck and dyspnea. The patient had no history of trauma or of having undergone any medical or odontological 


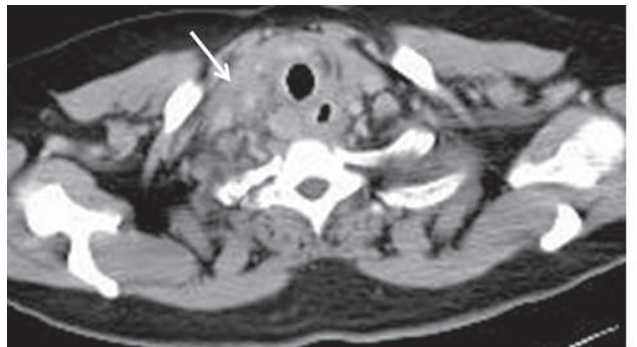

a

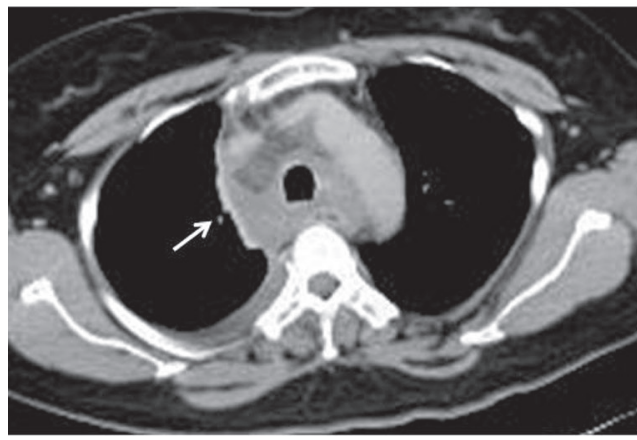

C

Fig. 1 A contrast-enhanced computed tomography scan showing a low-density lesion in the retropharynx and a high-density lesion in the right lobe of the thyroid gland (a; arrow). The low-density lesion is descending to the mediastinum at the level of bronchial bifurcation and a small amount of effusion is detected in the right pleura (b-d; arrows).

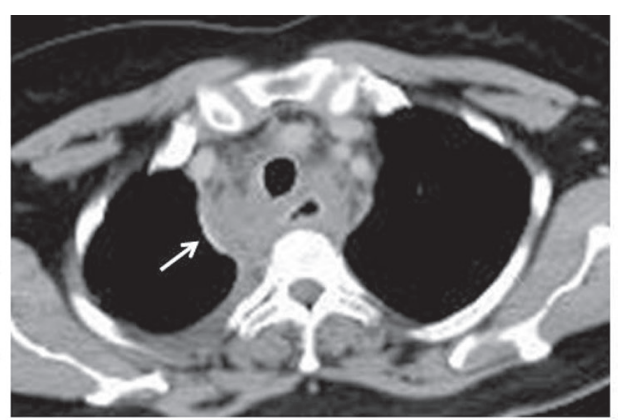

b

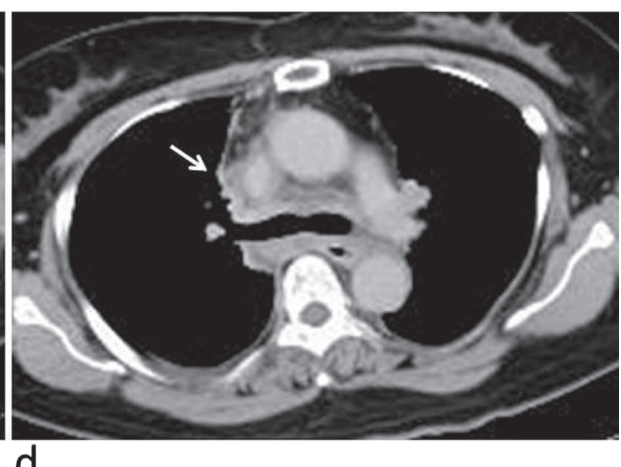

d procedures. A physical examination revealed diffuse swelling of the neck with slight tenderness. The patient's body temperature was $37.6^{\circ} \mathrm{C}$. An ultrasound (US) examination of the neck revealed the presence of an obscure mass located behind the right lobe of the thyroid gland. A chest X-ray showed widening of the upper mediastinum. A computed tomography (CT) scan indicated the presence of a low-density lesion extending from the retropharynx to the mediastinum and a high-density lesion located behind the right lobe of the thyroid gland, indicating active bleeding originating from either the thyroid or the parathyroid glands. A right pleural effusion was also observed, with a density close to that of blood. No gas bubbles were observed in the mediastinum (Fig. 1). The laboratory tests indicated high inflammatory reactions and remarkable anemia. The patient's coagulation function and bleeding times were both normal. Taken together, acute bleeding of the neck was suspected. The disease with which we were most concerned in making the differential diagnosis was descending necrotizing mediastinitis (DNM), an infectious disorder of high mortality. Our patient had clinical symptoms, i.e. neck swelling and fever, and clinical manifestations that are 


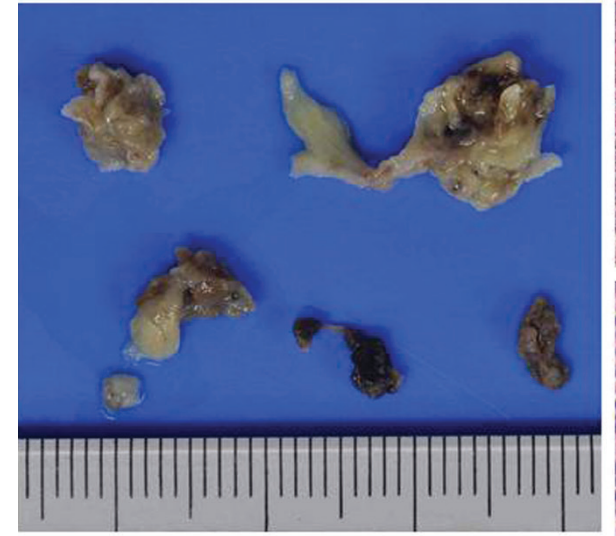

a

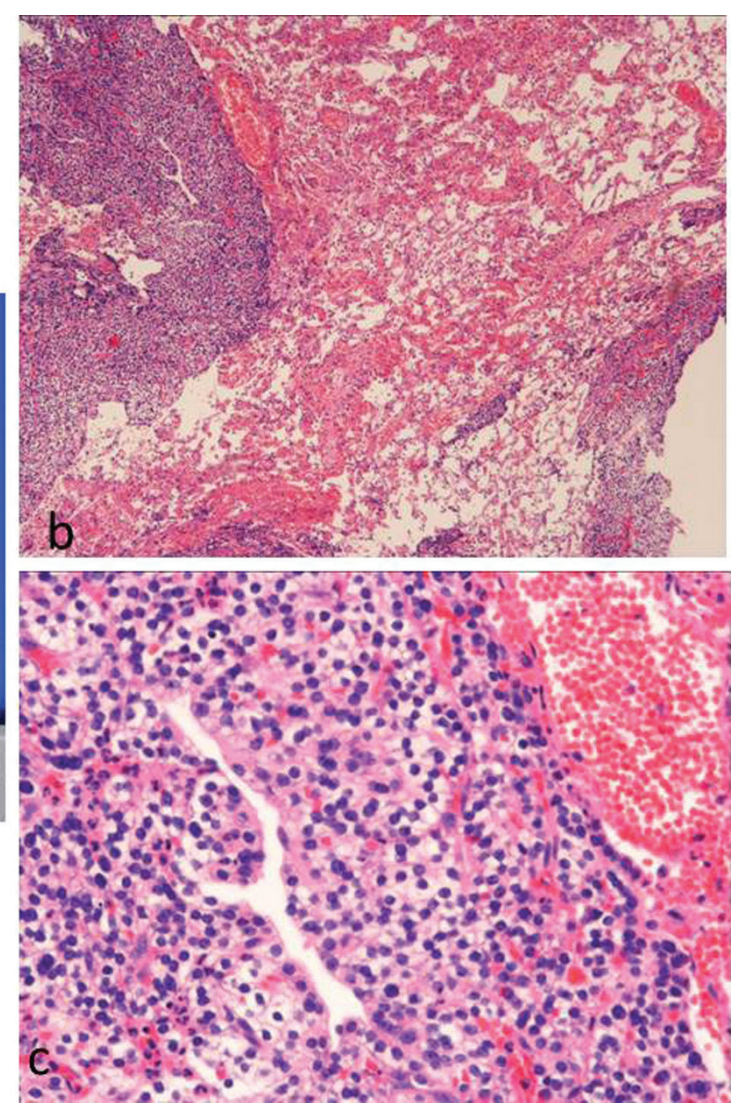

Fig. 2 A fragmented mass located behind the right lobe of the thyroid gland was found to be irregular and diffuse in macroscopic appearance (a). A parathyroid adenoma with acute hemorrhage. The parathyroid glands are considerably enlarged, showing increased cellularity and no stromal fat. Hematoxylin and eosin stains $(\mathbf{b}: \times 20 ; \mathbf{c}: \times 100)$. remaining parathyroid tissue. The patient was followed only by obtaining periodic blood exams.

\section{Surgical findings}

A right cervical approached was initiated with a vertical skin incision. We found the neck to be swollen due to a hematoma; however, we detected no abscesses. A fragmented mass located near the area behind the right lobe of the thyroid gland was removed, and oozing was found without active bleeding. Furthermore, thoracoscopy of the right chest revealed a bloody pleural effusion and an upper mediastinal hematoma. No abscesses were found in the operative field and no mediastinitis was observed.

\section{Histopathological findings}

The extirpated fragmented mass (six pieces) was found to be irregular and diffuse in macroscopic appearance (Fig. 2a). Microscopy revealed the mass to be a parathyroid adenoma with acute hemorrhage (Figs. 2b and 2c).

\section{Discussion}

Cervical and mediastinal hemorrhages occur mostly after trauma, injury or iatrogenic events, i.e. surgery, needle biopsy or catheterization of an artery in the neck or chest. Spontaneous cervical or mediastinal hemorrhages occur rarely. Over the last 80 years, approximately 20 cases of acute hemorrhage resulting from the spontaneous rupture of the parathyroid glands have been reported in the English literature. A previous study reported that the causes of these hemorrhages include parathyroid glands that are enlarged by adenomas, ${ }^{1-9)}$ primary or secondary hyperplasia, ${ }^{10-12)}$ cysts $^{13-15)}$ and carcinomas. ${ }^{16)}$ Some authors report that blood supplies may occasionally fail to meet the increased demands caused by these lesions. ${ }^{12)}$ Symptoms may persist from several days to one month or longer, and the tumors can cause life-threatening airway obstruction due to 
rapid bleeding. Hemorrhage in the intrathoracic parathyroid glands may cause the formation of a mediastinal hematoma; however, some of these events occur without neck manifestations. ${ }^{9)}$

Parathyroid adenomas typically draw clinical attention because patients show overproduction of PTH. However, some patients are asymptomatic and present with normal serum levels of PTH and calcium. ${ }^{3-5,7)}$ Because of its rarity, a spontaneous rupture of a parathyroid adenoma is difficult to detect in patients who lack a diagnosis of adenoma, hyperplasia or pathologies of the parathyroid glands. The first case ${ }^{1)}$ was diagnosed by autopsy. Rapidly progressing airway narrowing typically leads to death.

Such events are extremely difficult to diagnose preoperatively. Regarding treatment, the most important strategy is to reduce the pressure on the outer airways using drainage. Successful (or unexpected) extraction of the parathyroid gland lesion also assists in diagnosis.

Regarding the differential diagnosis in the present case, we were first concerned with a diagnosis of DNM. This disease is a rare and serious infection with a high mortality rate $[14 \%-50 \%]$ that originates from a pharyngeal or dental infection. ${ }^{17-21)}$ Our patient presented with symptoms similar to those of DNM. The patient's clinical symptoms included the gradual deterioration of neck pain, neck swelling and inflammatory reactions, and the tomographical manifestations included swelling of the retropharyngeal soft tissue, a low-density lesion located in the mediastinum and a pleural or pericardial effusion. However, the absence of gas bubbles in the mediastinum on CT scans was a key tomographic finding that changed the differential diagnosis from DNM. The surgical findings revealed an absence of abscesses and infection in the cervicomediastinal region, which are required to diagnose DNM.

No standardized follow-up is established for patients with acute cervicomediastinal hematomas. No recurrent cases have been reported. Such patients can be monitored by measuring serum hormone levels and monitoring for the emergence of any new pathologic entities in the remaining parathyroid tissue.

In summary, we herein reported a case of acute cervicomediastinal hematoma secondary to the spontaneous rupture of a parathyroid adenoma. When examining a patient who presents with acute swelling of the neck and a widened mediastinum without any preceding trauma or trigger, a diagnosis of acute cervicomediastinal hematoma should be considered. The purpose of treatment is to prevent airway compression caused by progressive hematomas. Such consideration is thus considered to lead to prompt and timely surgical intervention.

\section{Disclosure Statement}

The authors have no conflicts of interest to declare.

\section{References}

1) Capps RB. Multiple parathyroid tumors with massive mediastinal and subcutaneous hemorrhage. Am J Med Sci 1934; 188: 800-4.

2) Shundo Y, Nogimura H, Kita, Y, et al. Spontaneous parathyroid adenoma hemorrhage. Jpn J Thoracic Cardiovasc Surg 2002; 50: 391-4.

3) Kihara M, Yokomise H, Yamauchi A, et al. Spontaneous rupture of a parathyroid adenoma presenting as a massive cervical hemorrhage: report of a case. Surg Today 2001; 31: 222-4.

4) Shim WS, Kim IK, Yoo SD, et al. Non-functional parathyroid adenoma presenting as a massive cervical hematoma: a case report. Clin Exp Otorhinolaryngol 2008; 1: 46-8.

5) Hotes LS, Barzilay J, Cloud LP, et al. Spontaneous hematoma of a parathyroid adenoma. Am J Med Sci 1989; 297: 331-3.

6) Nito T, Miyajima C, Kimura M, et al. Parathyroid adenoma causing spontaneous cervical hematoma: a case report. Acta Otolaryngol Suppl 2007; 160-3.

7) Tonerini M, Orsitto E, Fratini L, et al. Cervical and mediastinal hematoma: presentation of an asymptomatic cervical parathyroid adenoma: case report and literature review. Emerg Radiol 2004; 10: 213-5.

8) Santos GH, Tseng CL, Frater RW. Ruptured intrathoracic parathyroid adenoma. Chest 1975; 68: 844-6.

9) Berry BE, Carpenter PC, Fulton RE, et al. Mediastinal hemorrhage from parathyroid adenoma simulating dissecting aneurysm. Arch Surg 1974; 108: 740-1.

10) Hedman I, Hansson G, Romanus R, et al. Massive parathyroid hemorrhage in a case of water-clear cell hyperplasia. Acta Chir Scand 1978; 144: 541-3.

11) Akimoto $T$, Saito $O$, Muto $S$, et al. A case of thoracic hemorrhage due to ectopic parathyroid hyperplasia with chronic renal failure. Am J Kidney Dis 2005; 45: 109-14.

12) Roma J, Carrió J, Pascual R, et al. Spontaneous parathyroid hemorrhage in a hemodialysis patient. Nephron 1985; 39: 66-7.

13) Dick JA, Brame KG, Owen WJ. Spontaneous bleeding into a parathyroid cyst. Br J Surg 1985; 72: 693.

14) Taniguchi I, Maeda T, Morimoto K, et al. Spontaneous retropharyngeal hematoma of a parathyroid cyst: report of a case. Surg Today 2003; 33: 354-7.

15) Simcic KJ, McDermott MT, Crawford GJ, et al. Massive extracapsular hemorrhage from a parathyroid cyst. Arch Surg 1989; 124: 1347-50. 
16) Erdas E, Licheri S, Lai ML, et al. [Cervico-mediastinal hematoma secondary to extracapsular hemorrhage of parathyroid carcinoma. Clinical case and review of the literature]. Chir Ital 2003; 55: 425-34.

17) Weaver E, Nguyen X, Brooks MA. Descending necrotising mediastinitis: two case reports and review of the literature. Eur Respir Rev 2010; 19: 141-9.

18) De Freitas RP, Fahy CP, Brooker DS, et al. Descending necrotising mediastinitis: a safe treatment algorithm. Eur Arch Otorhinolaryngol 2007; 264: 181-7.
19) Sancho LM, Minamoto H, Fernandez A, et al. Descending necrotizing mediastinitis: a retrospective surgical experience. Eur J Cardiothorac Surg 1999; 16: $200-5$.

20) Corsten MJ, Shamji FM, Odell PF, et al. Optimal treatment of descending necrotising mediastinitis. Thorax 1997; 52: 702-8.

21) Makeieff M, Gresillon N, Berthet JP, et al. Management of descending necrotizing mediastinitis. Laryngoscope 2004; 114: 772-5. 